\title{
Mining microsatellite markers from public expressed sequence tags databases for the study of threatened plants
}

\author{
Lua Lopez ${ }^{1,2}$, Rodolfo Barreiro ${ }^{2}$, Markus Fischer ${ }^{3}$ and Marcus A. Koch ${ }^{1 *}$
}

\begin{abstract}
Background: Simple Sequence Repeats (SSRs) are widely used in population genetic studies but their classical development is costly and time-consuming. The ever-increasing available DNA datasets generated by high-throughput techniques offer an inexpensive alternative for SSRs discovery. Expressed Sequence Tags (ESTs) have been widely used as SSR source for plants of economic relevance but their application to non-model species is still modest.

Methods: Here, we explored the use of publicly available ESTs (GenBank at the National Center for Biotechnology Information-NCBI) for SSRs development in non-model plants, focusing on genera listed by the International Union for the Conservation of Nature (IUCN). We also search two model genera with fully annotated genomes for EST-SSRS, Arabidopsis and Oryza, and used them as controls for genome distribution analyses. Overall, we downloaded 16031 555 sequences for 258 plant genera which were mined for SSRsand their primers with the help of QDD1. Genome distribution analyses in Oryza and Arabidopsis were done by blasting the sequences with SSR against the Oryza sativa and Arabidopsis thaliana reference genomes implemented in the Basal Local Alignment Tool (BLAST) of the NCBI website. Finally, we performed an empirical test to determine the performance of our EST-SSRs in a few individuals from four species of two eudicot genera, Trifolium and Centaurea.
\end{abstract}

Results: We explored a total of 14498726 EST sequences from the dbEST database (NCBI) in 257 plant genera from the IUCN Red List. We identify a very large number (17 102) of ready-to-test EST-SSRs in most plant genera (193) at no cost. Overall, dinucleotide and trinucleotide repeats were the prevalent types but the abundance of the various types of repeat differed between taxonomic groups. Control genomes revealed that trinucleotide repeats were mostly located in coding regions while dinucleotide repeats were largely associated with untranslated regions. Our results from the empirical test revealed considerable amplification success and transferability between congenerics.

Conclusions: The present work represents the first large-scale study developing SSRs by utilizing publicly accessible EST databases in threatened plants. Here we provide a very large number of ready-to-test EST-SSR (17 102) for 193 genera. The cross-species transferability suggests that the number of possible target species would be large. Since trinucleotide repeats are abundant and mainly linked to exons they might be useful in evolutionary and conservation studies. Altogether, our study highly supports the use of EST databases as an extremely affordable and fast alternative for SSR developing in threatened plants.

Keywords: Conservation, Evolution, EST-SSR, Functional markers, Population genetics, Threatened plants

\footnotetext{
* Correspondence: marcus.koch@cos.uni-heidelberg.de

${ }^{1}$ Center for Organismal Studies (COS) Heidelberg/Botanic Garden and

Herbarium Heidelberg (HEID), University of Heidelberg, Im Neuenheimer Feld

345, D-69120 Heidelberg, Germany

Full list of author information is available at the end of the article
}

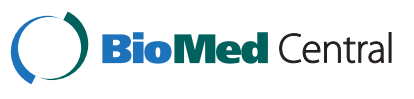

(C) 2015 Lopez et al. Open Access This article is distributed under the terms of the Creative Commons Attribution 4.0 International License (http://creativecommons.org/licenses/by/4.0/, which permits unrestricted use, distribution, and reproduction in any medium, provided you give appropriate credit to the original author(s) and the source, provide a link to the Creative Commons license, and indicate if changes were made. The Creative Commons Public Domain Dedication waiver (http://creativecommons.org/publicdomain/zero/1.0/) applies to the data made available in this article, unless otherwise stated. 


\section{Background}

Since the 1980s, the fast advent of molecular markers technology has revolutionized the field of genetics by changing the pace and accuracy of genetic analysis. Today, the analysis of DNA variation is a key component in plant genetics studies addressing relevant aspects such as evolution, phylogeny or conservation [1-3]. Among the various types of molecular markers used for these purposes, Simple Sequence Repeats (SSRs) are often regarded as the markers of choice because of their abundance, multiallelic behavior, high polymorphism and codominant inheritance [4]. Despite that the recent development of next generation sequencing (NGS) techniques has facilitated the de novo development of SSRs, this task is still quite expensive and requires a substantial amount of time [5]. Furthermore, genomic SSRs are usually species-specific, meaning that markers developed for one taxon are not always directly transferred to another [6]. In fact, the rates of successful cross-species transferability vary greatly between taxonomic groups [7].

With the recent and growing emphasis on functional genomics, the number of large datasets of DNA sequences generated by high-throughput technologies has largely increased for a wide variety of taxa. In this context, Expressed Sequence Tags (ESTs) databases available for public use arise as an attractive alternative for SSR mining and development [8-10]. Microsatellites generated from ESTs (i.e. EST-SSRs) display several advantages over those derived from anonymous DNA regions. First, time and costs for SSR development are considerably lower. Instead of the weeks required for SSRs development with conventional approaches, it takes 2-3 days to obtain a batch of EST-SSR markers, together with the primers needed for their testing, from existing databases. Second, any type of SSR motif can be detected in EST-SSR mining while a subset of predefined motifs are favored in conventional approaches which involve an enrichment cloning step. Third, SSRs have been found to be moderately abundant $(\approx 2-5 \%)$ also in EST sequences due to the preferential association with the non-repetitive fraction of the plant genome [11, 12]. Finally, EST-SSRs located in conserved regions are highly transferable between related species, and often even genera, because the conserved flanking sequences are ideally suited for primer design $[13,14]$. The latter facilitates comparisons among related taxa for addressing the mechanisms behind population diverge and speciation as well as comparisons among several co-occurring species $[15,16]$.

Nevertheless, EST-SSRs also pose some challenges. Their development is restricted to organisms with existing EST databases; although SSR mining from EST sequences of related species is also a promising alternative. In addition, EST-SSRs are expected to display lower levels of polymorphism than anonymous SSRs as they are associated with conserved regions of the genome $[9,17]$. Nonetheless, several studies with EST-SSRs found moderate to high level of polymorphism [18-20]. Finally, another possible concern regarding EST-SSRs is that these often non-neutral loci might bias the estimates of population divergence under the assumption of a neutral model of drift, mutation and migration [21]. However, several studies reported that population structure measures derived from EST-SSRs were consistent with those from anonymous SSRs, and as a matter of fact, only a very small fraction of all genes might have experienced recent positive selection [22-24].

EST-SSRs can be considered "functional markers" because ESTs represent a portion of the transcribed region of the genome under certain conditions [17, 25]. For a majority of these markers, a "putative function" can be deduced by comparison against annotated reference genomes. Dinucleotide repeats in ESTs are known to be favored in Untranslated Regions (UTRs) and introns, while trinucleotide repeats are frequently associated to coding regions [12]. Thus, compared with anonymous microsatellites, EST-SSRs offer the opportunity to detect variation in the transcribed portion of the genome that could show a marker-trait association [17].

To date, EST-SSR markers have been successfully used for resolving phylogenies [26] and to increase resolution in comparative genetic mapping studies by cross-referencing genes between species [13, 27]. These studies have been mainly focused on species with economic relevance and model species [11, 28-31]. Even if EST-SSRs can be regarded as a potential tool for addressing evolutionary and conservation-related questions in threatened plant species, their application in these type of studies has been overlooked and examples in the literature are limited [20,32].

The present study explores the development of markers from public EST databases for evolutionary and conservation genetic studies of non-model plants, with special emphasis in threatened species. We searched all plant genera included in the International Union for the Conservation of Nature (IUCN) Plant Red List which had EST sequences available in the dbEST database (GenBank at the National Center for Biotechnology Information-NCBI). Since most of these plant genera do not include model organisms, normally there are no available annotated reference genomes for comparison, hampering the location of the EST-SSRs within the genome. Since the location of the EST-SSRs across the different regions in the genome might have implications in the analysis and interpretation of the results, we analyzed in depth the EST sequences data sets for two model genera with well-known annotated genomes and used them as a proxy. By doing so, we aimed to identify general distribution patterns of the various types of repeats and motifs along the different regions of the genome that can be applied for the remaining analyzed genera. The 
genus Arabidopsis was selected as a control for Eudicotyledoneae while Oryza was used as a guide for Monocotyledoneae. Finally, a proof-of-concept study was undertaken by testing for amplification, cross-amplification and polymorphism of 24. EST-SSRs in four species from two genera (Trifolium fragiferum L., Trifolium saxatile All., Centaurea valesiaca (DC.) Jord and Centaurea borjae Valdés-Bermejo \& Rivas Goday). These four species are of conservation interest due to their threatened status: T. saxatile is listed as near threatened [33] and C. borjae as endangered [34] by the IUCN, while $T$. fragiferum is catalogued as vulnerable and $C$. valesiaca as near threatened in the Swiss Red List of endangered vascular plants [35].

\section{Methods}

\section{Sequence data sources}

By September 2013, 16031555 sequences were downloaded from the dbEST database in GenBank at the NCBI website (http://www.ncbi.nlm.nih.gov/dbEST/). Batch files of EST sequences were downloaded in FASTA format. The dataset included 14498726 records for 257 genera (Oryza included) listed both in IUCN Red List and dbEST plus 1532829 records for Arabidopsis.

\section{EST-SSRs detection and primer design}

SSRs were detected in the EST datasets with the help of QDD1 [36]. Before SSR search, QDD1 assembled the ESTs of each genus into unique sequences (contigs and singletons) to avoid redundancy. Non-redundant EST sequences were then screened for perfect SSRs. In the present study only Class I microsatellites, defined as DNA sequences containing at least $20 \mathrm{bp}$, were considered [37]. That is ten repeats for di-, seven for tri-, five for tetra- and four for penta- and hexanucleotide repeats respectively. Mononucleotide repeats were excluded from the EST-SSRs search as their polymorphism is often difficult to interpret. To have enough flanking sequence of appropriate quality for primer design, only EST sequences larger than 100 bp were taken into account during EST-SSR searches. EST-SSR primers were designed with the version of Primer3 embedded in QDD1 [38] under the following criteria: length of primers ranging from 18-23 nucleotides (optimum $20 \mathrm{bp}$ ), annealing temperature $55-65{ }^{\circ} \mathrm{C}$ (optimum $60{ }^{\circ} \mathrm{C}$ ), GC content $30-70 \%$ (optimum $50 \%$ ) and PCR product size from 90 to $320 \mathrm{bp}$.

\section{Basal local alignment search tool (BLAST) searches in Oryza and Arabidopsis}

EST sequences for the control genera, Oryza and Arabidopsis, were run in QDD1 following the criteria specified above. QDD1 output files were then used as inputs for the BLASTn search against Oryza sativa and
Arabidopsis thaliana reference genomes using default parameters specified on the NCBI website. Whenever a positive hit was found, the matching gene sequence was downloaded and aligned in Geneious 6.1.6 (created by Biomatters, available from http://www.geneious.com/). The distribution of the SSRs towards the genome (i.e. UTRs, exons, introns, genomic regions) was inferred using the annotated gene information derived from the BLASTn search. As double-check, a BLASTx search against the Oryza and Arabidopsis reference protein databases was also conducted for EST-SSRs using the megablast option with the default algorithm parameters.

\section{Compositional analysis of SSR mining}

Occurrence and frequency of SSR motifs in the IUCN genera were analyzed after importing QDD1 output files into MATLAB and Statistics Toolbox 2013a (MathWorks Inc., MA, US) (Additional file 1). Repeat types, number of repeats, and frequency were calculated for each genus using a combination of sorting and counting functions. Results were displayed using tabular and graphical representations. To provide a broader view, results from the IUCN genera were grouped in eight taxonomic groups: Florideophyceae, Charophyceae, Monilophyta, Lycopodiophyta, Acrogymnospermae, Magnoliidae, Monocotyledoneae and Eudicotyledoneae [39].

\section{DNA isolation, PCR conditions, and amplification of SSRs}

Six individuals of Trifolium fragiferum, seven from Centaurea valesiaca, two individuals of Trifolium saxatile and two from Centaurea borjae were used for the screening of EST-SSR amplification. Fresh leaves were dried in silica gel until DNA extraction. Leaf tissue from each plant was collected in a $2.0 \mathrm{ml}$ Eppendorf tube, frozen with liquid nitrogen and ground to fine powder with a MiniBeadBeater (Glen Mills Inc, NJ, US). DNA was extracted using the Wizard Magnetic Kit (Promega, Madison, WI, US) according to manufacturer instructions. The quality of the extracted DNA and negative controls were checked in $1.5 \%$ agarose gels. Twelve primer pairs were selected for each genus to test the EST-SSRs amplification. Amplification was tested with a standard PCR reaction performed in $25 \mu \mathrm{l}$ containing $1 \mathrm{x}$ reaction buffer (NzyTech, Lisboa, Portugal), $2 \mathrm{mM} \mathrm{MgCl}_{2}$ (NzyTech), $0.2 \mu \mathrm{M}$ of each dNTP (Fermentas GmBH, St. Leon-Rot, Germany), $0.16 \mu \mathrm{M}$ of each primer, $1 \mu \mathrm{l}$ of genomic DNA and 0.5 units of DNA polymerase (NzyTech). PCR profiles consisted of 5 min denaturation at $94{ }^{\circ} \mathrm{C}$ followed by 35 cycles of $30 \mathrm{~s}$ denaturation at $94{ }^{\circ} \mathrm{C}, 50 \mathrm{~s}$ annealing at $59{ }^{\circ} \mathrm{C}$, $45 \mathrm{~s}$ of extension at $72{ }^{\circ} \mathrm{C}$, with a final elongation step of 35 min at $72{ }^{\circ} \mathrm{C}$. PCR products were screened on $2 \%$ agarose gels. Primer pairs that had successfully amplified in the first round where re-tested with the M13 tail method [40]. PCR reactions were performed following the 
procedure specified above with the addition of $0.04 \mu \mathrm{M}$ of the forward primer with the M13 tail and $0.16 \mu \mathrm{M}$ of the reverse and $0.16 \mu \mathrm{M}$ of the M13-FAM primer. PCR profiles comprised 5 min denaturation at $94{ }^{\circ} \mathrm{C}$ followed by 35 cycles of $30 \mathrm{~s}$ denaturation at $94{ }^{\circ} \mathrm{C}, 50 \mathrm{~s}$ annealing at $59{ }^{\circ} \mathrm{C}$, and $45 \mathrm{~s}$ of extension at $72{ }^{\circ} \mathrm{C}$, followed by eight additional cycles of $30 \mathrm{~s}$ denaturation at $94{ }^{\circ} \mathrm{C}$, $45 \mathrm{~s}$ annealing at $53{ }^{\circ} \mathrm{C}, 45 \mathrm{~s}$ of extension at $72{ }^{\circ} \mathrm{C}$, and a final elongation step of $35 \mathrm{~min}$ at $72{ }^{\circ} \mathrm{C}$. PCR products were screened on $2 \%$ agarose gels and sized on an ABI3730XL DNA analyzer (Applied Biosystems, Foster City, CA, US) using a 500HD size ladder. PCR reactions from

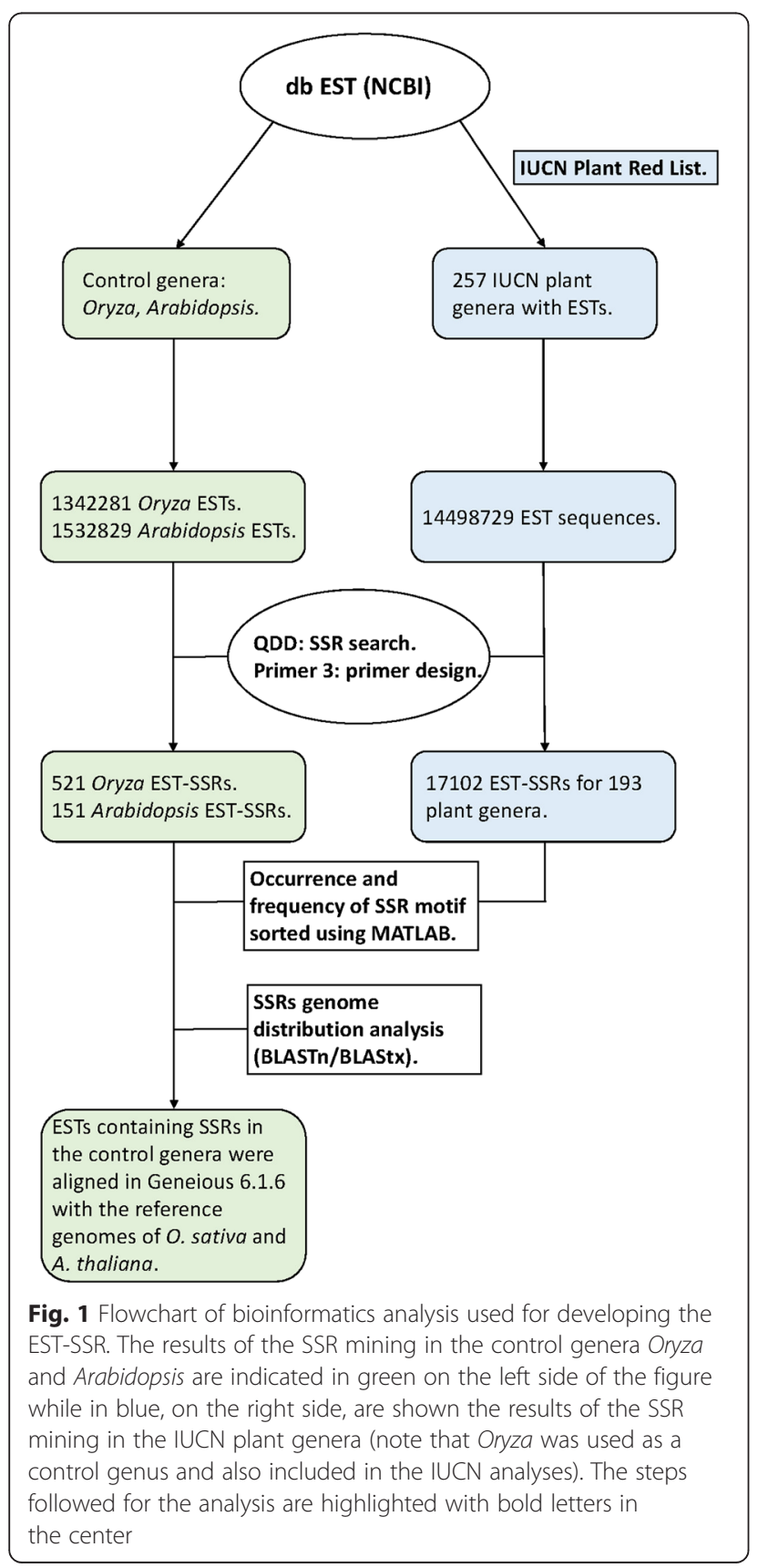

one primer pair that produced PCR amplicons larger than expected were purified with $1 \mu \mathrm{l}$ of Exonuclease I $(20 \mathrm{u} /$ $\mu \mathrm{l})($ Fermentas $\mathrm{GmBH})$ and $2 \mu \mathrm{l}$ of FastAP $(10 \mathrm{u} / \mu \mathrm{l})($ Fermentas $\mathrm{GmBH}$ and bi-directionally sequenced (BigDye Terminator cycling conditions) in an Automatic Sequencer 3730XL (Applied Biosystems).

\section{Results}

\section{Frequency and distribution of SSRs in Arabidopsis and Oryza}

The dbEST database contained 1342281 Oryza EST sequences (Fig. 1). After filtering redundant sequences and those shorter than $100 \mathrm{bp}, 2626$ EST sequences (1 912 singletons and 714 contigs) remained for the microsatellite and primer search. From those, QDD1 found 521 perfect EST-SSRs with primer pairs (19.2\%). On the other hand, the Arabidopsis dataset encompassed 1532 829 EST sequences that, after filtering, was reduced to 899 EST sequences (616 singletons and 283 contigs) that contained 151 perfect microsatellites with primer pairs (16.8 \%) (Fig. 1). In both cases, filtering had a large impact on the number of EST records available for SSR search, indicating a high rate of redundant and/or short records in the EST database.

Although only sequences assigned to Oryza were downloaded from the dbEST, $23.8 \%$ of the analyzed EST sequences containing SSRs did not render a significant hit in the BLASTn search against the Oryza sativa reference genome. Similarly, the BLASTn comparison of Arabidopsis EST sequences containing SSRs against the Arabidopsis thaliana reference genome had $8 \%$ of unsuccessful searches. The microsatellites derived from these sequences were excluded from further analysis. Thus, the distribution and position of 397 and 139 ESTSSRs were determined for Oryza and Arabidopsis, respectively (Table 1). Trinucleotide repeats were the commonest repeat size in both genera with very similar relative abundances $(61.96 \%$ in Oryza and $69.78 \%$ in Arabidopsis). Dinucleotide repeats were second in abundance (23.29 \% in Oryza and $17.27 \%$ in Arabidopsis), while tetra- and pentanucleotide repeats were scarce in both genera $(<5 \%)$. Hexanucleotide repeats displayed intermediate frequencies in both genera $(11.59 \%$ in Oryza and $8.63 \%$ in Arabidopsis).

The various SSR motifs were grouped into classes according to base complementarity and depending on the reading frame (for groups see Fig. 2; from now on, in the text they will be identified with the first motif repeat). Motifs in dinucleotide repeats displayed similar patterns in both genera as the AG group was the most abundant while the AC and AT groups were scarce, and those from the GC group went undetected (Fig. 2). Despite that the AG group prevailed in both genera, it was clearly commoner in Oryza than in Arabidopsis. Unlike 
Table 1 Number and distribution of the EST-SSRs found for the EST sequences of Oryza and Arabidopsis

\begin{tabular}{|c|c|c|c|c|c|c|c|c|c|c|}
\hline & Genomic & Intron & UTR & Exon & Total & & & & & \\
\hline & Oryza & Arabidopsis & Oryza & Arabidopsis & Oryza & Arabidopsis & Oryza & Arabidopsis & Oryza & Arabidopsis \\
\hline Dinucleotides & 17 & 2 & 26 & 5 & 29 & 16 & 1 & 1 & 73 & 24 \\
\hline Trinucleotides & 18 & 2 & 16 & 2 & 70 & 26 & 142 & 67 & 246 & 97 \\
\hline Tetranucleotides & 3 & 0 & 3 & 0 & 9 & 3 & 2 & 0 & 17 & 3 \\
\hline Pentanucleotides & 4 & 0 & 1 & 0 & 10 & 3 & 0 & 0 & 15 & 3 \\
\hline Hexanucleotides & 6 & 1 & 3 & 0 & 13 & 1 & 24 & 10 & 46 & 12 \\
\hline Total & 48 & 5 & 49 & 7 & 131 & 49 & 169 & 78 & 397 & 139 \\
\hline
\end{tabular}

SSR search was only carried out in those EST sequences downloaded from the dbEST database (NCBI) that had a match in their respective reference genomes using BLASTn

dinucleotide, trinucleotide repeats displayed different patterns in each genus. Various motifs from trinucleotide repeats which were found in Oryza, went unrecorded (GGC and ACG) or very rare (AGC, ACC and AGG) in Arabidopsis. Likewise, the AAC group found in Arabidopsis was absent in Oryza. The group GGC dominated in Oryza, while motifs from the AAG, AGC and AGG groups had intermediate values, and the AAT group was clearly underrepresented (Fig. 2). In comparison, trinucleotide repeats in Arabidopsis were dominated by the AAG group, while AGC and AAT groups were very scarce (i.e. only one and two SSR detected, respectively). No motif of the ATG group was found for either genera.
Using the alignments of the EST sequences containing SSRs and the reference genomes downloaded from GenBank, four possible location categories were established based on to the information provided by the BLASTn results: introns, untranslated regions (UTRs), exons and genomic region (when the EST-SSR did not fall in any of the other categories). Overall, the majority of EST-SSRs were located in exons $(42.57 \%$ in Oryza and $56.12 \%$ in Arabidopsis) followed by UTRs (33.00\% in Oryza and $35.25 \%$ in Arabidopsis) and only a small fraction of the EST-SSRs was found in introns (12.34 \% in Oryza and $5.04 \%$ in Arabidopsis) and genomic regions (12.09\% in Oryza and $3.60 \%$ in Arabidopsis)

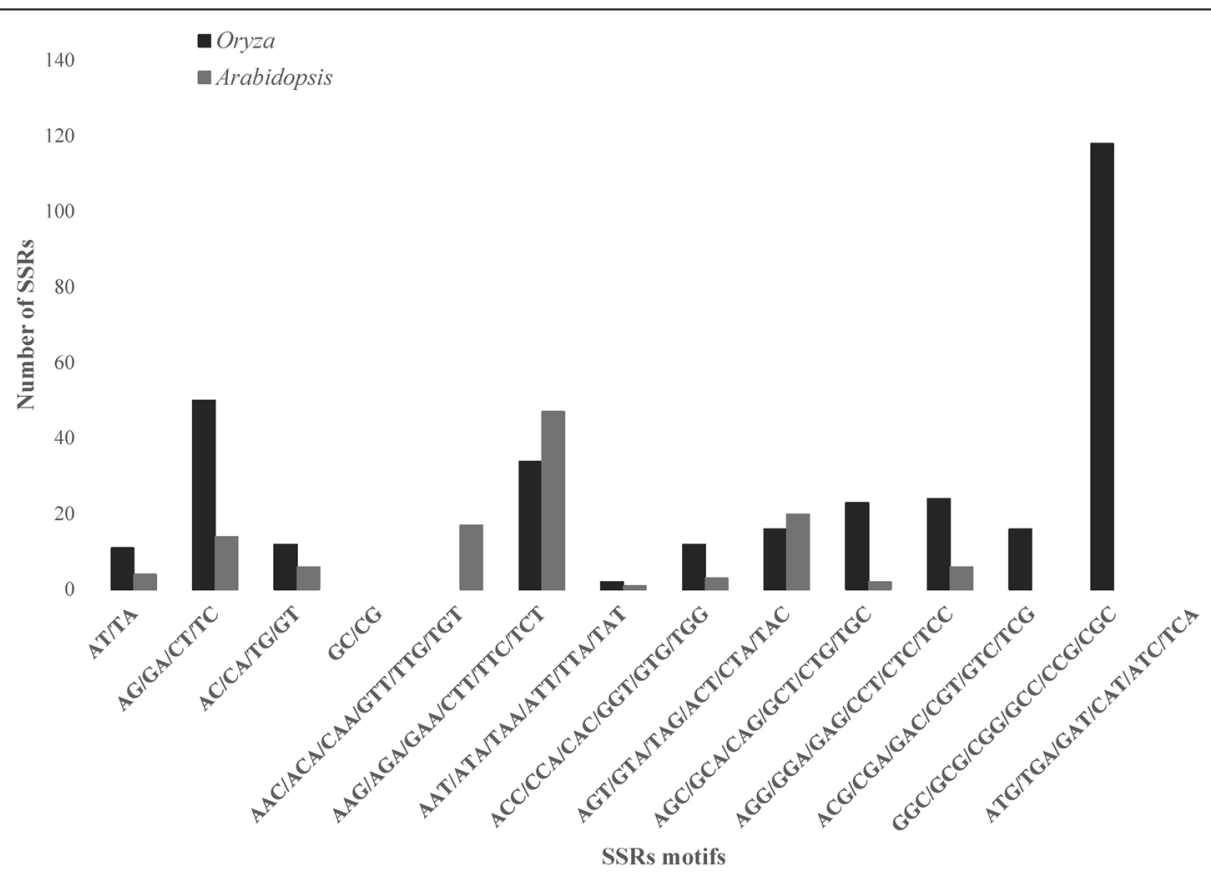

Fig. 2 Di- and trinucleotides distribution obtained using QDD1 software from Oryza and Arabidopsis EST sequences that had a positive hit in the Oryza sativa (japonica cultivar-group) and Arabidopsis thaliana reference genomes database with BLASTn (NCBI). Oryza is represented in black while Arabidopsis is displayed in grey. The different types of motif are detailed in axis X while the number of SSRs for each class are showed in axis $Y$ 
(Table 1). The proportion of EST-SSRs found in introns and genomic regions in Oryza was more than two and four times larger than in Arabidopsis, as it would be expected due to the compact and small genome of Arabidopsis. Repeats of different size showed characteristic locations along the genome. Thus, tri- and hexanucleotide repeats were mostly concentrated in exons in both genera $(57.72 \%$ and $69.07 \%$ of the total number of trinucleotide repeats in Oryza and Arabidopsis, and $52.17 \%$ and $83.33 \%$ of the total number of hexanucleotide repeats in Oryza and Arabidopsis). In contrast, dinucleotide repeats mostly occurred in non-coding regions, mainly in UTRs (39.73\% of the total dinucleotide repeats in Oryza and $66.67 \%$ in Arabidopsis) but also in introns (35.62\% of the total dinucleotide repeats in Oryza and 20.83 \% in Arabidopsis) and genomic regions (23.29\% of the total dinucleotide repeats in Oryza and $8.33 \%$ in Arabidopsis). Tetra- and pentanucleotide repeats were scarce and they occurred almost only associated to non-coding regions (except for $11.76 \%$ of Oryza's pentanucleotide repeats which were located in exons).

\section{EST-SSRs analysis from the IUCN genera}

Two hundred and fifty-seven genera included in the IUCN plant red list were mined for SSR using the EST sequences available in the dbEST (NCBI) (Fig. 1). These genera included two Florideophyceae, one Charophyceae, three Lycopodiophyta, five Monilophyta, five Magnoliidae, 18 Acrogymnospermae, 58 Monocotyledoneae and 165 Eudicotyledoneae. Overall, 14498726 sequences were screened for SSR discovery (Table 2). In a few cases, SSR search and primer design were unsuccessful due to a very low number of EST sequences in the input file or sequences that did not fulfill the predefined criteria. As a result, 193 genera were successfully mined for SSRs rendering 17102 microsatellites with their respective primers (see Additional file 2: Table S1). From the total number of EST-SSR, the largest proportion belonged to Eudicotyledoneae covering $73.19 \%$, followed by Monocotyledoneae (18.17 \%) and Acrogymnospermae (8.29\%) while each of the remaining groups had $<1 \%$ frequency. The percentage of SSR found was related with the number of EST sequences downloaded, for example, the group Eudicotyledoneae represented $67.19 \%$ of the total number of EST sequences and the Monocotyledoneae $22.05 \%$. Nevertheless, the latter is not true for the Acrogymnospermae where the number of EST sequences analyzed were $8.20 \%$ and the frequency of EST-SSR was $3.33 \%$.

As in the control genomes, di- and trinucleotide repeats were the commonest types of SSR $(30.76 \%$ and $39.03 \%$ respectively) while tetra- and pentanucleotide repeats were very scarce $(6.76 \%$ and $7.06 \%$ respectively), and hexanucleotide repeats displayed an intermediate position (16.38\%). Nonetheless, when the frequency of the various classes of SSR was analyzed in detail, there were differences among taxonomic groups (Fig. 3). Trinucleotide repeats were commoner than dinucleotide repeats in eudicots (38.50\% vs. $33.23 \%)$ and monocots (44.88 \% vs. $19.24 \%$ ). In Acrogymnospermae, hexanucleotide repeats dominated representing more than one third of the SSRs followed by di- and trinucleotide repeats with a $25 \%$ frequency each. Furthermore, trinucleotide repeats prevailed in Lycopodiophyta $(64.21 \%)$, while dinucleotide repeats dominated in Monilophyta (81.65 \%) and Magnoliidae (58.13\%). In Florideophyceae tri- and hexanucleotide repeats displayed the highest frequencies (40\% each type of repeat). Finally, tetra- and pentanucleotide repeats were rare across all groups ( $\leq 10 \%$ each type) except in Charophyceae where each one represented almost $20 \%$ of the total.

Overall, the most abundant dinucleotide repeats were from the AG group. For trinucleotide repeats there was no consensus along all the groups studied, but overall the AGT and AGC groups were the commonest. When each taxonomic group was considered separately, the AT group was very common in Acrogymnospermae while in red algae the ACG and GGC groups were the most frequent. Moreover, the GC-rich trinucleotide repeats displayed high abundance in Monocotyledoneae while they were absent from the remaining groups. Tetra-, penta- and hexanucleotide repeats were too scarce in most taxa to allow an appropriate analysis of their distribution.

\section{Amplification and transferability of the EST-SSRs}

A subset of 24 pairs of EST-SSR primers (12 pairs per genus) were chosen to test amplification performance in two genera of Eudicotyledoneae, Trifolium and Centaurea (Table 3). A total of 53422 Trifolium EST sequences were run for SSR search. The ESTs data set of Trifolium included three species Trifolium pretense L., Trifolium repens L. and Trifolium purpureum Loisel, with 38 109, 15 260 and 53 EST sequences respectively. The SSR search rendered 130 EST-SSRs with their primers; 23 were di-, 77 tri-, 11 tetra-, 9 penta- and 10 hexanucleotide repeats (Additional file 2: Table S1). From those, 12 EST-SSRs were selected; three di-, seven tri- and two tetranucleotide repeats. Six of the selected EST-SSRs derived from unique sequences of $T$. repens and four from unique sequences of T. pratense, while the two remaining derived from contigs of $T$. pratense (Table 3). Likewise, the 85293 EST sequences analyzed for Centaurea comprehended two Centaurea species, Centaurea maculosa Lam. and Centaurea sosltitialiis L. with 44886 and 40407 EST sequences respectively. The Centaurea EST sequences returned 306 EST-SSRs with their primers; 24 were di-, 146 tri-, 33 tetra-, 26 penta- repeats and 77 hexanucleotide repeats. From those, 12 EST-SSRs were selected; three di-, six tri-, two tetra- and one hexanucleotide 
Table 2 Number of ETS-SSRs found in the IUCN plant genera containing EST sequences in the dbEST

\begin{tabular}{|c|c|c|c|c|c|c|c|c|c|c|}
\hline Taxonomic groups & $\mathrm{Ng}$ & $\mathrm{Ng}$ SSR & $N_{\text {EST }}$ & Dinucleotides & Trinucleotides & Tetranucleotides & Pentanucleotides & Hexanucleotides & Total & Commonest motifs \\
\hline Florideophyceae & 2 & 2 & 16645 & 2 & 10 & 2 & 1 & 10 & 25 & ACG/GGC \\
\hline Charophyceae & 1 & 1 & 88280 & 16 & 77 & 39 & 38 & 30 & 200 & $\mathrm{AG} / \mathrm{TGA}$ \\
\hline Acrogymnospermae & 18 & 15 & 1191184 & 144 & 145 & 30 & 58 & 193 & 570 & AG/AT/CAG \\
\hline Lycopodiophyta & 3 & 3 & 101292 & 20 & 122 & 15 & 7 & 26 & 190 & $\mathrm{AG} / \mathrm{CAG} / \mathrm{TGA}$ \\
\hline Monilophyta & 5 & 3 & 35665 & 129 & 18 & 3 & 2 & 6 & 158 & $\mathrm{AG} / \mathrm{TGA}$ \\
\hline Magnoliidae & 5 & 5 & 68569 & 193 & 89 & 11 & 9 & 30 & 332 & AG/AT/CAG \\
\hline Monocotyledoneae & 58 & 37 & 3197142 & 598 & 1395 & 296 & 323 & 496 & 3108 & AG/AT/AAG/CGG \\
\hline Eudicotyledoneae & 165 & 127 & 9742277 & 4160 & 4820 & 760 & 769 & 2010 & 12519 & AG/AT/AAG/TGA \\
\hline Total & 257 & 193 & 14498726 & 5262 & 6676 & 1156 & 1207 & 2801 & 17102 & \\
\hline
\end{tabular}

$\mathrm{Ng}$ number of genera, $\mathrm{Ng} \mathrm{SSR}_{\mathrm{R}} \mathrm{N}_{E S T}$ number of EST sequences downloaded, number of genera with SSRS 


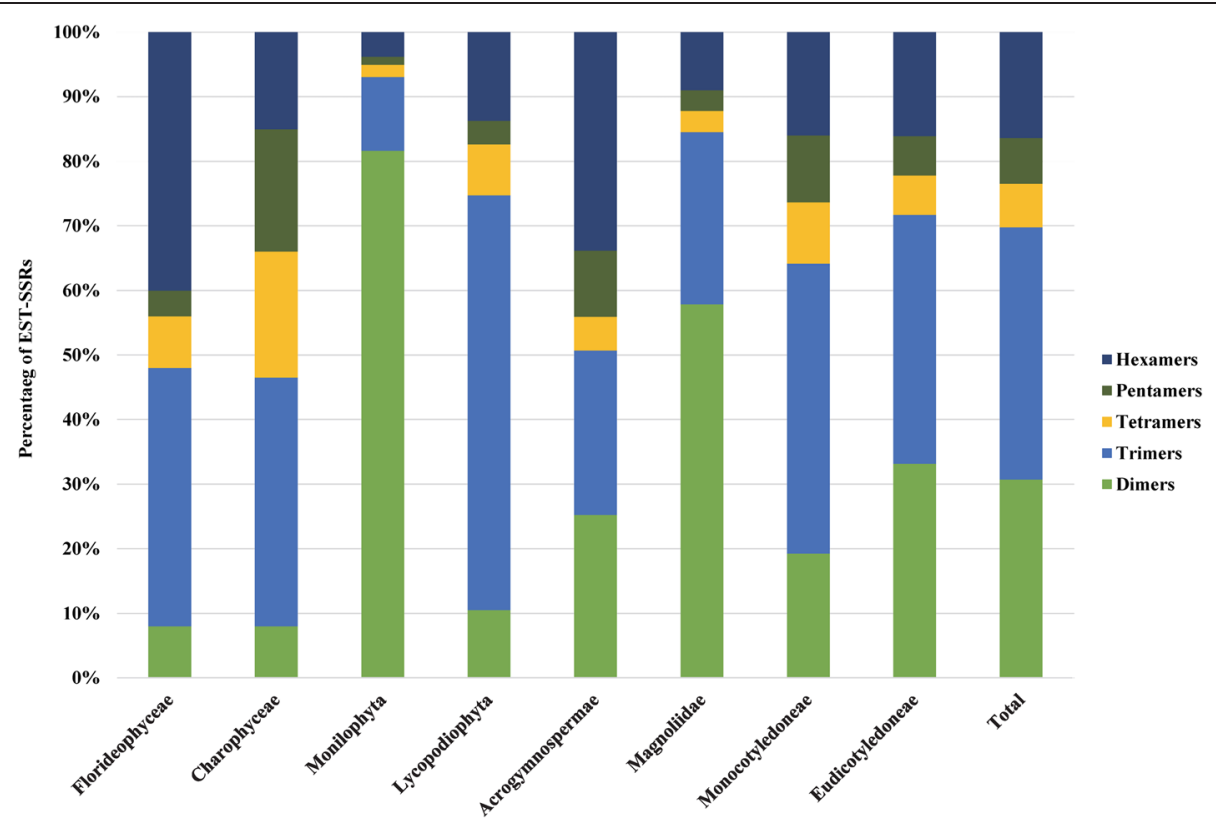

Fig. 3 Distribution of EST-SSRs in 193 plant genera including threatened species by the IUCN. Bars in the X axis represent each taxonomic group investigated and the whole dataset. The axis Y represents the percentage of EST-SSRs found within each group. Colors in each bar indicate the type of repeat: dinucleotide repeats in light green, trinucleotide repeats in light blue, tetranucleotide repeats in yellow, pentanucleotide repeats in dark green and hexanucleotide repeats in dark blue

repeat. Five of the selected EST-SSRs derived from unique sequences of $C$. solstitialis and five from unique sequences of C. maculosa, while the two remaining derived from contigs of C. maculosa and C. solstitialis respectively (Table 3). Overall, thirteen out of the 24 pairs of primers yielded a clear amplification product (amplification rate $54.2 \%)$. Nevertheless, the amplification success differed between genera and Centaurea displayed a higher amplification rate (eight out of 12 EST-SSRs, $58.3 \%$ ) than Trifolium (five out of 12 EST-SSRs, $41.7 \%$ ).

All loci produced amplification products of the expected size, except for locus C6 of Centaurea that generated an amplicon longer than expected, suggesting the presence of a non-transcribed intron inside; which was further confirmed by sequencing the PCR product. The M13-tail protocol had mostly no impact on PCR performance since all pairs of primers that amplified in the unmodified state (first round of amplification) were also functional with an M13-tail attached. However, locus C7 produced a larger unspecific second band with the M13tail method.

Despite the small number of individuals used in the empirical test, three out of the seven EST-SSRs that yielded a PCR product of the expected size in Centaurea displayed polymorphism in the seven individuals of Centaurea valesiaca (loci $\mathrm{C} 1, \mathrm{C} 7$ and $\mathrm{C} 11$ produced two, two and three genotypes) while for the two individuals of Centaurea borjae loci C7, C9 and C11 were polymorphic. Four loci, C4, C7, C9 and C11, showed variability between Centaurea species (Table 4). On the other hand, one of the dinucleotide repeats of Trifolium (loci T1) displayed a stutter-peak profile and was discarded from further analysis. Among the four remaining loci T5 displayed polymorphism in Trifolium fragiterum while loci $\mathrm{T} 5$ and $\mathrm{T} 9$ were polymorphic in Trifolium saxatile. Two loci, T6 and T9, showed variability between species (Table 4).

The selected primers were also used to assess the cross-species transferability in Centaurea and Trifolium (Table 4). Cross-species transferability is considered successful when the EST-SSR is functional (i.e. one or two PCR product are present) and it is polymorphic (i.e. two or more alleles in the genera) [7]. Almost all the loci fulfilled the aforementioned criteria (75\%). All ESTSSRs that worked in one species were functional in its counterpart but three loci, C6 and C2 in Centaurea and loci T4 in Trifolium, were not polymorphic for the genera as only one allele was detected (Table 3 ).

\section{Discussion}

Computational approaches allow the fast discovery of molecular markers from the ever-increasing publicly available genomic resources. Thus, SSRs derived from EST sequences arise as an excellent alternative to the classical techniques based on anonymous microsatellites because of their fast and inexpensive discovery [9]. Besides, unlike anonymous SSRs, EST-SSRs markers have been proven of great value in cross-species studies, 
Table 3 EST-SSRs tested empirically in two Eudicotyledoneae genera, Trifolium and Centaurea

\begin{tabular}{|c|c|c|c|c|c|c|c|c|}
\hline \multirow[t]{2}{*}{ Locus } & \multirow[t]{2}{*}{ GenBank accession No. } & \multirow[t]{2}{*}{ Species } & \multicolumn{2}{|l|}{ Primer sequences } & \multirow{2}{*}{$\begin{array}{l}\text { Repeat } \\
\text { motif }\end{array}$} & \multirow{2}{*}{$\begin{array}{l}\text { Expected } \\
\text { size (bp) }\end{array}$} & \multirow[t]{2}{*}{$N_{\mathrm{A}}$} & \multirow[t]{2}{*}{ Size range (bp) } \\
\hline & & & Forward & Reverse & & & & \\
\hline \multirow[t]{2}{*}{ T6-Trifolium } & gi86106666 & T. pratense & CAACCAGTGGTGTGAGTAGGAG & ACGTTGGTGGAGAGGTTGAG & $(A G)_{11}$ & $110-128$ & 2 & $114-116$ \\
\hline & gi86105378 & & & & & & & \\
\hline T7-Trifolium & gi428283538 & T. repens & ATCACGCTTCACTCCTCCAC & CAACTCCAAGCTTAAGATCGTGTA & $(A G)_{13}$ & $110-122$ & & no $P C R$ product \\
\hline T1-Trifolium & gi428292074 & T. repens & AGATTCCCACCAATCTCCCT & CAATACGCGGGTCTTGATCT & $(A G)_{11}$ & $210-228$ & --- & $257-261$ \\
\hline \multirow[t]{2}{*}{ T2-Trifolium } & gi86106666 & T. pratense & TTCCGGTTAGGTTAGGGTTT & TITCACATCTTCCGAAGCC & $(\mathrm{AAT})_{7}$ & $110-113$ & & no PCR product \\
\hline & gi86105378 & & & & & & & \\
\hline T3-Trifolium & gi428285635 & T. repens & CACCACATATGCAACCACAA & GTCGACGACGGTTGTTACCT & $(\mathrm{AGT})_{8}$ & $110-126$ & & no $P C R$ product \\
\hline T8-Trifolium & gi428291122 & T. repens & GCAAAACTCAAGAGAACGGC & GGATGTCTTCGGAGGTGAGA & $(\mathrm{ACC})_{7}$ & $110-122$ & & no $P C R$ product \\
\hline T9-Trifolium & gi428292435 & T. repens & ACAACCCATTTGCCTCAAAG & ТТTСАСТТССАССАССТСС & $(\mathrm{ACC})_{7}$ & $110-133$ & 2 & $124-127$ \\
\hline T10-Trifolium & gi86119186 & T. pratense & TCCACTAGTTCTAGAGCGGC & TCCTGTAAACTGGAGGAGCC & $(\mathrm{ACC})_{9}$ & $110-153$ & & no PCR product \\
\hline T11-Trifolium & gi86124411 & T. pratense & TGGCGGTGGTGACTTATACA & TGTTGGGAGTGGTGATGTT & $(A G G)_{8}$ & $110-153$ & & no PCR product \\
\hline T4-Trifolium & gi86125686 & T. pratense & GCTGCCACAGCACTACCAG & AATATTACCGTGAATGAAGCTCAG & $(\mathrm{ACC})_{8}$ & $110-113$ & 1 & 110 \\
\hline T5-Trifolium & gi86097190 & T. pratense & TGAGTTCCGAGTTAAGGCTCA & TTCGGTAACTCCGAGGATTG & $(\mathrm{ACCT})_{5}$ & $210-217$ & 2 & $227-230$ \\
\hline T12-Trifolium & gi428282514 & T. repens & GATTATTCAACCAAACGCCG & TAGAAAGCCACGCCAAGACT & $(\text { AATCC) })_{20}$ & 290 & & no PCR product \\
\hline C6-Centaurea & gi124618051 & C. maculosa & TGGGATGCAGTCCAGTCATA & TTGCAACTTGCCTGTACCAC & $(A C)_{11}$ & $160-162$ & 1 & 256 \\
\hline C1-Centaurea & gi148298213 & C. maculosa & GGGAACCACACCTITCATCT & GATCTGGCTTGACCCAAGAA & $(\mathrm{AC})_{10}$ & $90-119$ & 2 & 99-101 \\
\hline C7-Centaurea & gi124669731gi124688599 & C. solstitialis & TCGTTITCCGATCACAAACTC & CAATTTGGCGACATCTCCTT & $(A C)_{12}$ & $110-160$ & 4 & $114-152$ \\
\hline C2-Centaurea & gi124680442 & C. solstitialis & CGCATTATGGAATAAACCCG & GCTTTCGACTTCATAAGCGG & $(\mathrm{AAG})_{7}$ & $140-152$ & 1 & 147 \\
\hline C8-Centaurea & gi148296795 & C. maculosa & CGATGTATACAGGTGGTGCG & GGAGAAGGGGAGACGTAAGG & $(\mathrm{ACC})_{7}$ & $110-150$ & 2 & $141-144$ \\
\hline C9-Centaurea & gi124675484 & C. solstitialis & AACGGTAGGAACCAGCATTG & GATCCTCTGGCAGGGTCATA & $(\mathrm{ACC})_{9}$ & $260-302$ & 4 & $290-299$ \\
\hline C10-Centaurea & gi124661102 & C. solstitialis & AGTTGCCAGAAAGGAGCAAG & TCGAGAACAATGGCCTATCC & $(\mathrm{AGC})_{7}$ & $210-229$ & & no $P C R$ product \\
\hline C11-Centaurea & gi148292432 & C. maculosa & TCCATGGATACAACCACCAA & GCGATATTCGGATGCAAAGT & $(A G G)_{7}$ & $160-175$ & 4 & $160-172$ \\
\hline C3-Centaurea & gi124632630 & C. maculosa & GCCATCCCCTTCTCTACTCC & GTTACAGGTGACGATGGGG & $(\mathrm{AGT})_{7}$ & $160-181$ & & no PCR product \\
\hline C4-Centaurea & gi124691992 & C. solstitialis & CTGCACCTACCCAGAGAAGC & CGGGAGAGGGTAAATTGTGA & $(\mathrm{AGGT})_{5}$ & $110-115$ & 3 & $103-109$ \\
\hline C12-Centaurea & gi124632477 & C. maculosa & ATGCATTGAGAAGGCCAATC & AACTCGCAAGCCTITTCAAG & $(\mathrm{AATCGG})_{4}$ & $210-223$ & & no PCR product \\
\hline \multirow[t]{3}{*}{ C5-Centaurea } & gi1 24673348 & C. solstitialis & TTAAGCATTCTTCGAGGCGT & TCTATGCCTACGCCGATCTC & $(\mathrm{AAGCAG})_{5}$ & 110 & & no PCR product \\
\hline & gi124676118 & & & & & & & \\
\hline & gi1 24669484 & & & & & & & \\
\hline
\end{tabular}

GenBank accession No., identification number of the EST sequences (when more than one ID refers to consensus sequence); species, indicates the species of the EST sequences; primer sequences; type of repeated motif; expected size of the PCR product; $N_{\mathrm{A}}$, number of alleles for the examined individuals and size range of the PCR product (-- indicates stutter peak) 
Table 4 Cross-species transferability of EST-SSRs in two plant genera, Trifolium and Centaurea

\begin{tabular}{|c|c|c|c|c|}
\hline Locus & $N_{\mathrm{A}}$ & Size Range (bp) & $N_{A}$ & Size Range (bp) \\
\hline & \multicolumn{2}{|c|}{ Centaurea valesiaca $(n=7)$} & \multicolumn{2}{|c|}{ Centaurea borjae $(n=2)$} \\
\hline C6-Centaurea & 1 & 256 & 1 & 256 \\
\hline C1-Centaurea & 2 & 99-101 & 2 & $90-101$ \\
\hline C7-Centaurea & 2 & $141-143$ & 4 & $114-152$ \\
\hline C2-Centaurea & 1 & 305 & 1 & 305 \\
\hline C8-Centaurea & 1 & 144 & 1 & 141 \\
\hline C9-Centaurea & 1 & 290 & 3 & $293-299$ \\
\hline C11-Centaurea & 2 & $160-166$ & 3 & $160-169$ \\
\hline \multirow[t]{2}{*}{ C4-Centaurea } & 1 & 103 & 2 & $105-109$ \\
\hline & \multicolumn{2}{|c|}{ Trifolium fragiterum $(n=6)$} & \multicolumn{2}{|c|}{ Trifolium saxatile $(n=2)$} \\
\hline T6-Trifolium & 1 & 114 & 1 & 116 \\
\hline T1-Trifolium & -- & $257-261$ & -- & $257-261$ \\
\hline T9-Trifolium & 1 & 124 & 2 & $124-127$ \\
\hline T4-Trifolium & 1 & 110 & 1 & 110 \\
\hline T5-Trifolium & 2 & $227-230$ & 2 & $227-230$ \\
\hline
\end{tabular}

$n$ number of individuals tested, $N_{A}$ number of alleles for the examined individuals and size range of the PCR product (-- indicates stutter peak)

linkage maps and discovering markers linked to genes [13]. So far, EST-SSRs have mainly targeted crop and model species [11, 29-31]. In contrast, the use of ESTSSRs in evolutionary and conservation studies with nonmodel species are still scarce [20, 32]. In this context, the present study has tried to fill this gap by providing EST-SSRs for plant genera listed by the IUCN which can be applied immediately in evolutionary and conservation genetic studies in a very large number of threatened species.

\section{Frequency and distribution of SSRs in Arabidopsis and Oryza}

The frequency and distribution of microsatellites in EST sequences is highly variable among studies, in part because the efficiency of SSR discovery relies on several factors such as the mining tool used, the mining criteria, or the size of the EST sequences dataset $[29,41]$. Differences in the mining criteria usually lead to significant deviations in the number of microsatellites identified in a given species using the same dataset [29]. Here, we opted for a conservative criteria and only Type I microsatellites were considered in an effort to increase the polymorphism of the detected ETS-SSRs [41]. As a consequence, we probably obtained a lower number of EST-SSRs than would have been found if more relaxed parameters had been set for the searching.

The in-depth analysis of the EST-SSRs frequency and their distribution in Arabidopsis and Oryza revealed that tri- and dinucleotide repeats encompassed more than $85 \%$ of the total SSRs found. Furthermore, trinucleotide repeats comprehended the vast majority of the SSRs. High frequencies of trinucleotide repeats are known to be favored in higher plants and have been invariably reported in most studies $[11,24]$. As expected in vascular plants, the AG group were the most abundant dinucleotide repeat motif and low frequencies of the group AT were recorded in both genera [11, 12, 24, 37]. In agreement with previous studies of monocots and dicots, we found differences in the trinucleotide repeats of Oryza and Arabidopsis. GC-rich motifs, commonly dominant in monocots, were the most frequent trinucleotide repeats in Oryza as the group GGC [11, 24, 28, 37] while the AAG group prevailed in Arabidopsis and GCrich motifs were absent [24].

Overall, a major fraction of EST-SSRs were located in exons, an observation that seems consistent with ESTSSRs deriving from transcribed regions. Nevertheless, not every type of nucleotide repeat appeared in exons with equal probability. Di, tetra and pentanucleotide repeats were mostly concentrated in UTRs and, to a lesser extent, in other non-coding regions, whereas triand hexanucleotide repeats regularly occurred in exons. Since the frequency and distribution of the different SSR repeats and their motifs are function of the dynamics and history of genome evolution, the predominance of trinucleotide repeats in ESTs is attributed to selection against frameshift mutations caused by length variation in non-trinucleotide repeat motifs [12]. Large frequencies of dinucleotide repeats in UTRs and the prevalence of trinucleotide repeats in exons have been consistently reported in plant studies [28, 42]. Since EST sequences are derived from mRNA, the frequency of EST-SSRs located in non-coding regions might seem higher than expected. However, transcripts of unknown function with apparently little protein coding capacity are known to overlap with protein-coding regions and they are often distributed in intergenic regions [43].

Interestingly, trinucleotide repeats in Oryza were rich in GC motifs and more than $70 \%$ of these GC-rich trinucleotides were related to exons. CCG repeats have been found to be involved in many gene functions as stress resistance, transcription regulation, or metabolic enzyme biosynthesis [28]. Trinucleotide repeats usually involve a moderate number of repeats because they do not perturb the reading frame but they may alter the stability of the quaternary structure of the resulting protein; this may result in low levels of polymorphism [44]. In contrast, dinucleotide repeats tend to display higher levels of variation as consequence of their association with UTRs and other non-coding regions $[27,45]$.

\section{EST-SSRs analysis from the IUCN genera}

Overall, the frequencies of the various nucleotide repeats and motifs in IUCN genera were highly consistent with 
the results derived from the control genomes Oryza and Arabidopsis. Tri- and dinucleotide repeats accounted for more than $60 \%$ of the total EST-SSRs, while tetra-, penta- and hexanucleotide repeats displayed lower frequencies. However, the abundance of the various types of repeat differed between groups. The latter, was expected because the SSRs distribution is a function of the dynamics and history of genome evolution [12]. Results from monocots and eudicots were highly consistent with the two control genomes and with previous findings in flowering plants where trinucleotide repeats were the most abundant motifs followed by dinucleotide repeats [24]. Similarly, the AG group was the commonest dinucleotide repeat, as it is typically the case in angiosperms $[11,12,24,37,46]$. The pattern seen in the trinucleotide motifs of IUCN genera agreed with what we found in Oryza and Arabidopsis, corroborating the high abundance of CG-rich motifs in monocots and the AAG group in dicots [11, 24, 28, 37]. In line with earlier studies, Acrogymnospermae revealed a higher proportion of hexanucleotide repeats, as well as dinucleotide repeats from the AT group when compared with monocots and eudicots [24, 46, 47]. Unfortunately, the four groups of non-vascular plants were represented by too few genera to allow generalizations.

Finally, in some genera (e.g. Taiwania and Urochloa) no Type I SSR was detected despite that the number of EST sequences in the data set seemed enough (2 624 and 2207 respectively). The latter might be a consequence of the conservative criteria use in this study in an effort to increase the polymorphism of the detected ETS-SSRs [41]. To test the impact of the mining criteria a second SSR search, using more relaxed parameters, was carried out in the 64 genera with no Type I SSR and in ten randomly selected genera with Type I SSR. The SSR discovery was done using the default parameter suggested by QDD1, which is at least four repeats for each type of perfect SSRs. By doing so, four out of the 64 genera rendered SSRs, but only one SSR each one. Thus, it seems that in most of the cases the absence of output for the SSR search was largely caused by the filtering parameters instead of the searching criteria, indicating high rates of redundancy and/or short sequences in the input file. However, when the same test was performed in the ten randomly selected genera with Type I SSR the impact of relaxing the searching parameters was larger (see Additional file 3: Table S2). The number of ESTSSRs detected increased an average of $67.79 \%$, ranging from $34.78 \%$ till $116 \%$. As expected, the higher impact was in di- and trinucleotide repeats. Therefore, in those cases when the number of EST sequences for the pet species is not very large, or the number of Type I SSR is too small, the parameters for SSR mining can be relaxed allowing the detection of a larger number of markers.

\section{Amplification and transferability of the EST-SSRs}

Amplification success in this study was similar to values reported in some studies of EST-SSRs [48, 49] but lower than others $[50,51]$. Unsuccessful primer amplification can be a consequence of non-transcribed introns located in the primer region [41]. Also, some of the EST-SSRs detected in our searches could actually belong to a different organism. As revealed by the analysis of Arabidopsis and Oryza, a portion of EST sequences did not find a match in their annotated genomes and might be a result of RNA contamination [17].

Given their association with conserved genome regions, EST-SSRs are often assumed to be less polymorphic than their genomic counterparts $[9,17,52]$. However, studies comparing both types of markers showed that this premise does not always hold true and similar levels of polymorphism have been found in anonymous SSRs versus EST-SSRs [18, 19]. Since only few individuals of each genus were selected to test the performance of our EST-SSRs, the levels of polymorphism detected in this study cannot be considered a general attribute of EST-SSRs. Saying so, our EST-SSRs showed acceptable levels of polymorphism within species, as well as divergence between species. The quality of the banding patterns was high, with clear peaks (except for the T1 pair), a flat baseline, and no null allele was detected. Cleaner profiles and lower frequencies of null alleles than those found with anonymous SSRs appear to be a general property of EST-SSRs $[22,51]$. The lower levels of polymorphism usually attributed to ESTSSRs compared with anonymous SSRs may be compensated by their high rate of cross-species transferability $[19,29,51]$, which has been reported not only among congenerics, but also across species of different genera [13]. Our results are highly congruent with the premise of high-transferability in EST-SSRs as all of the tested primers that successfully amplified in one species did the same in its counterpart and most of the loci displayed two or more alleles for the genera. Consequently, EST-SSRs arise as molecular markers with great potential for comparative studies among species.

\section{Use of EST-SSRs as molecular markers for studying threatened species}

EST-SSRs can be used for essentially the same purposes as genomic SSRs but their link to translated regions offers a range of possibilities not usually available in anonymous SSRs. The function of EST-SSRs linked to coding regions can be identified by comparison with protein databases, with annotated genomes of closely related species or with model organisms such as Arabidopsis for eudicots and Oryza for monocots. By doing so, researchers interested in threatened species can go one step further in their studies and infer levels 
of functional genetic diversity $[17,25]$. This topic has been largely disregarded due to the absence of well annotated genomes in non-model species like is the case in most threatened plant species. Therefore, the use of EST-SSRs for population studies will facilitate overcoming this issue. Furthermore, these markers can also be used in phylogenetic studies [26] and in comparative mapping studies thanks to their high cross-species transferability $[13,14]$.

It is often warned that population structure parameters estimated with EST-SSRs loci must be interpreted with caution as they may display a signature of selection [19]. However, this behavior is advantageous in studies targeting the so called "adaptive variation", a topic of high relevance in conservation studies [1]. So far, studies trying to identify signatures of selection relied on the detection of outlier loci using putatively neutral markers. As mentioned before, EST-SSRs are associated with the transcribed region of the genome, thus they have a higher probability to be under selective pressures. Besides, for those markers showing a sign of selection a putative function can be deduced by comparing the sequence containing the SSR against publicly available databases as the non-redundant protein database from the NCBI. Interestingly, several studies reported that population structure measures derived from EST-SSRs were in agreement with those from anonymous SSRs, and only a small fraction of all genes might have experienced recent positive selection [22-24]. Therefore, the variation in the degree of neutrality of EST-SSRs allows to choose appropriate markers for several types of studies, from neutral and near-neutral loci for estimates of genetic drift or gene flow, to non-neutral ones for studying selection-related questions.

Our results derived from the control genomes suggest that conservation studies with an aim on functional variation and/or interested in detecting signatures of selection should focus on trinucleotide repeats because they are highly likely to be located within exons and are more abundant and more polymorphic than hexanucleotide repeats. Although dinucleotide repeats are mainly linked to non-coding regions and they are expected to behave as neutral markers they should not be rule out from conservation studies because they are known to be very polymorphic and our results show that they are mainly linked to UTRs, which are known to be involved in gene expression and other control functions [53]. Overall, we would recommend that, when using EST-SSRs, di- and trinucleotide repeats should be combined for a more comprehensive approach. This way trinucleotide repeats would cover the direct link with exons displaying a higher probability of being subjected to selection processes while dinucleotide repeats would offer larger levels of polymorphism and their probable neutral behavior will facilitate the inference of population structure measures non-biased by selection. Moreover, since all EST-SSRs are associated with the transcribed region of the genome they can be used to target functional genetic diversity in threatened species. Besides, EST sequences containing SSRs can be cross-referenced with annotated genomes for sequence similarity and gene discovery.

\section{Conclusions}

In summary, this study represents the first large-scale attempt to assess the potential of publicly accessible EST databases as a source for SSRs discovery in threatened plants. Our results highly support the use of existing EST databases for SSRs discovery in non-model plants as a bench tool for evolutionary and/or conservation studies of geneticists and molecular ecologists. With this approach, we identified a very large number of ready-totest EST-SSRs in most of the IUCN plant genera used in this study. Our tests indicate that these SSRs can show high transferability rate among species. Therefore, the set of loci presented here possibly has a very large number of potential target species. Moreover, a portion of our loci might be functional markers providing relevant information about "adaptive variation", which is a subject of high interest in conservation studies. In fact, the variation in the degree of neutrality of EST-SSRs allows to select markers that may be appropriate for various research topics. Developing molecular markers for the species of interest is one of the most frequent ratelimiting steps in population genetic studies. In this regard, our results show that EST databases are a valuable and suitable source for SSRs discovery. Unlike the demanding classical procedure for genomic SSR development, a set of EST-SSRs with primers can be produced in a couple of days at no additional cost once the EST database has been accessed.

\section{Availability of supporting data}

The data sets supporting the results of this article (i.e. all the EST-SSRs, with their respective primers, developed in the present study for 193 plant genera from the IUCN Red List and for Arabidopsis) are publicly available in the Dryad database with doi:10.5061/dryad.63h33 (instructions for the database in Additional file 4).

\section{Additional files}

Additional file 1: Programming scripts. (DOCX 20 kb)

Additional file 2: Table S1. List of the IUCN plant genera mined for EST-SSRs with raw results. Number of species for each genera within each IUCN category ( $E X=$ extinct, $E W=$ extinct in the wild, $C R=$ critically endangered, $\mathrm{EN}=$ endangered, $\mathrm{VU}=$ vulnerable, $\mathrm{NT}=$ near threatened, LC = least concern, DD =data deficient). N EST, number of EST sequences 
downloaded for each genera; di-, tri-, tetra-, penta- and hexa- denotes type of SSRs and total indicates the total number of SSRs with primer information found for each genera. (DOCX $52 \mathrm{~kb}$ )

Additional file 3: Table S2. Impact of the mining criteria (Type I or relaxed parameters) in the total number of EST-SSRs detected in ten randomly selected genera. (DOCX $13 \mathrm{~kb}$ )

Additional file 4: Instructions for the database use. (DOCX $147 \mathrm{~kb}$ )

\section{Abbreviations}

EST: Expressed Sequence Tags; SSR: Simple Sequence Repeats; EST-SSR: SSR mined form EST sequences; NGS: Next Generation Sequencing;

IUCN: International Union for the Conservation of the Nature; NCBI: National Center for Biotechnology Information; BLAST: Basic Local Alignment Search Tool; UTR: untranslated region.

\section{Competing interests}

The authors declare that they have no competing interests.

\section{Authors' contributions}

LL, MK, RB designed the study. LL collected and analyzed the data. LL drafted the manuscript and MK, MF, and RB contributed to the final paper. All authors read and approved the final manuscript.

\section{Acknowledgments}

We thank Anne Kempel for providing tissue samples and Lisa Kretz, Maria Rodriguez Lojo, Eva Wolf, Florian Michling and Nora Hohmann for their technical help during the experimental phase. We acknowledge the financial support of the Deutsche Forschungsgemeinschaft and Ruprecht-Karls-Universität Heidelberg within the funding program Open Access Publishing. This research was supported by the European Science Foundation (ConGenOmics Network) and the University of A Coruña (Contratos Predoutorais UDC 2012).

\section{Author details}

${ }^{1}$ Center for Organismal Studies (COS) Heidelberg/Botanic Garden and Herbarium Heidelberg (HEID), University of Heidelberg, Im Neuenheimer Feld 345, D-69120 Heidelberg, Germany. ${ }^{2}$ BioCost research group, Departamento de bioloxía animal, vexetal e ecoloxía, Facultade de Ciencias, University of A Coruña, E-15008 A Coruña, Spain. ${ }^{3}$ Institute of Plant Sciences, University of Bern, Altenbergrain 21, CH-3013 Bern, Switzerland.

\section{Received: 25 February 2015 Accepted: 9 October 2015}

\section{Published online: 13 October 2015}

\section{References}

1. Frankham R, Briscoe DA, Ballou JD. Introduction to Conservation Genetics. 2nd ed. Cambridge: Cambridge University Press; 2010.

2. Höglund J. Evolutionary conservation genetics. Oxford: Oxford University Press; 2009.

3. Allendorf FW, Luikart G. Conservation and the Genetics of Populations. 2nd ed. Malden: Blackwell Pub.; 2012.

4. Ritland K. Marker-inferred relatedness as a tool for detecting heritability in nature. Mol Ecol. 2000;9:1195-204.

5. Squirrell J, Hollingsworth PM, Woodhead M, Russell J, Lowe AJ, Gibby M, et al. How much effort is required to isolate nuclear microsatellites from plants? Mol Ecol. 2003;12:1339-48.

6. Selkoe KA, Toonen RJ. Microsatellites for ecologists, a practical guide to using and evaluating microsatellite markers. Ecol Lett. 2006;9:615-29.

7. Barbará T, Palma-Silva C, Paggi GM, Bered F, Fay MF, Lexer C. Cross-species transfer of nuclear microsatellites markers: potential and limitations. Mol Ecol. 2007;16:3759-67.

8. Rudd S. Expressed Sequence Tags, alternative or complement to whole genome sequences? Trends Plant Sci. 2003;8:321-9.

9. Ellis JR, Burke JM. EST-SSRs as a resource for population genetic analyses. Heredity. 2007;99:125-32.

10. Zalapa JE, Cuevas C, Zhu H, Steffan S, Senalik D, Zeldin E, et al. Using next-generation sequencing approaches to isolate simple sequence repeat (SSR) loci in the plant sciences. Am J Bot. 2012;99:193-208.

11. Kantety RV, La Rota M, Matthews DE, Sorrells ME. Data mining for simple sequence repeats in expressed sequence tags from barley, maize, rice, sorghum and wheat. Plant Mol Biol. 2002;48:501-10.
12. Morgante M, Hanafey M, Powell W. Microsatellites are preferentially associated with nonrepetitive DNA in plant genomes. Nat Genet. 2002;30:194-200.

13. Varshney RK, Sigmund R, Börner A, Korzun V, Stein N, Sorrells ME, et al. Interspecific transferability and comparative mapping of barley EST-SSR markers in wheat, rye and rice. Plant Sci. 2005;168:195-202.

14. Jewell MC, Frere CH, Prentis PJ, Lambrides CJ, Godwin ID. Characterization and multiplexing of EST-SSR primers in Cynodon (Poaceae) species. Am J Bot. 2010;97:e99-e101.

15. Noor MAF, Feder JL. Speciation genetics: evolving approaches. Nat Rev Genet. 2006;403:851-61.

16. Whithman TG, Bailey JK, Schweitzer JA, Shuster SM, Bangert RK, LeRoy CJ, et al. A framework for community and ecosystem genetics: from genes to ecosystems. Nat Rev Genet. 2006;7:510-23.

17. Varshney RK, Graner A, Sorrells ME. Genic microsatellite markers in plants, features and applications. Trends Biotechnol. 2005;23:48-55.

18. Fraser LG, Harvey CF, Crowhurst RN, De Silva HN. EST-derived microsatellites from Actinidia species and their potential for mapping. Theor App Genet. 2004;108:1010-6.

19. Pashley CH, Ellis JR, McCauley DE, Burke JM. EST Databases as a source for molecular markers, lessons from Helianthus. J Hered. 2006:97:381-8.

20. Aleksic MA, Geburek T. Quaternary population dynamics of an endemic conifer, Picea omorika, and their conservation implications. Conserv Genet. 2014;15:87-107.

21. Luikart G, England PR, Tallmon DA, Jordan S, Taberlet P. The power and promise of population genomics, from genotyping to genome typing. Nat Rev Genet. 2003;4:981-94.

22. Woodhead M, Russel J, Squirrell J, Hollingsworth PM, Mackenzie K, Gibby M, et al. Comparative analysis of population genetic structure in Anthyrium distentifolium (Ptedidophyta) using AFLPs and SSRs from anonymous and trasncribed gene regions. Mol Ecol. 2005;14:1681-95.

23. Tiffin P, Hahn MW. Coding sequence divergence between two closely related plant species, Arabidopsis thaliana and Brassica rapa spp. pekinensis. J Mol Evol. 2002;54:746-53.

24. Victoria FC, Da Maia LC, De Oliveira AC. In silico comparative analysis of SSR markers in plants. BMC Plant Biol. 2011;11:15-29.

25. Andersen JR, Lübberstedt T. Functional markers in plants. Trends Plant Sci. 2003:8:554-60.

26. Tabbasam N, Zafar Y. Mehboob-ur-Rahman. Pros and cons of using genomic SSRs and EST-SSRs for resolving phylogeny of the genus Gossypium. Plant Syst Evol. 2013;300:559-75.

27. Yu JK, Dake TM, Singh S, Benscher D, Li W, Gill B, et al. Development and mapping of EST-derived simple sequence repeat markers for hexaploid wheat. Genome. 2004;47:805-18.

28. Gao L, Tang J, Li H, Jia J. Analysis of microsatellites in major crops assessed by computational and experimental approaches. Mol Breeding. 2003;12:245-61.

29. Aggarwal RK, Hendre PS, Varshney RK, Bhat PR, Krishakumar V, Singh L. Identification, characterization and utilization of EST-derived genic microsatellite markers for genome analyses of coffee and related species. Theor App Genet. 2007;114:359-72.

30. Simko I. Development of EST-SSR Markers for the study of population structure in Lettuce (Lactuca sativa L.). J Hered. 2009;100:256-62.

31. Fukuoka H, Yamaguchi H, Nunome T, Negoro S, Miyatake K, Ohyama A. Accumulation, functional annotation, and comparative analysis of expressed sequence tags in eggplant Solanum melongena L., the third pole of the genus Solanum species after tomato and potato. Gene. 2010;450:76-84.

32. Wöhrmann T, Guicking D, Khoshbakht K, Weising K. Genetic variability in wild populations of Prunus divaricata Ledeb. in northern Iran evaluated by EST-SSR and genomic SSR marker analysis. Genet Res Crop Evol. 2011;58:1157-67.

33. Gygax A, Montagnani C, Gargano D, Bernhardt KG, Gigot G. Trifolium saxatil. In: IUCN 2014. IUCN Red List of Threatened Species. 2013.

34. Gómez-Orellana Rodríguez L. Centaurea borjae. In: IUCN 2014. IUCN Red List of Threatened Species. 2013.

35. Moser DM, Gygax A, Bäumler B, Wyler N, Palese R. Rote Liste der gefährdeten Arten der Schweiz: Farn- und Blütenpflanzen. Bern; Zentrum des Datenverbundnetzes der Schweizer Flora, Chambésy; Conservatoire et Jardin botaniques de la Ville de Genève, Chambésy; BUWAL-Reihe «Vollzug Umwelt»; 2002.

36. Meglecz E, Costedoat C, Dubut V, Gilles A, Malausa T, Pech N, et al. QDD, a user-friendly program to select microsatellite markers and design primers from large sequencing projects. Bioinformatics. 2010;26:403-4. 
37. Temnykh S, DeClerck G, Lukashova A, Lipovich L, Cartinhour S, McCouch SR. Computational and experimental analysis of microsatellites in rice (Oryza sativa L.), frequency, length variation, transposon associations, and genetic marker potential. Genome Res. 2001;11:1441-52.

38. Rozen S, Skaletsky H. Primer3 on the WWW for general users and for biologist programmers. Methods Mol Biol. 2000;132:365-86.

39. Ruhfel BR, Gitzendanner MA, Soltis PS, Soltis DE, Burleigh JG. From algae to angiosperms-inferring the phylogeny of green plants (Viridiplantae) from 360 plastid genomes. BMC Evol Biol. 2014;14. doi:10.1186/1471-2148-14-23.

40. Schuelke M. An economic method for the fluorescent labeling of PCR fragments. Nat Biotechnol. 2000;18:233-4.

41. Blair MW, Hurtado N. EST-SSR markers from five sequenced cDNA libraries of common bean Phaseolus vulgaris L. comparing three bioinformatic algorithms. Mol Ecol Res. 2013;13:688-95.

42. Wang Z, Weber JL, Zhong G, Tanksley SD. Survey of plant short tandem DNA repeats. Theor App Genet. 1994;88:1-6.

43. Gingeras TR. Origin of phenotypes, genes and transcripts. Genome Res. 2007;17:682-90.

44. Cho GY, Ishii T, Temnykh S, Chen X, Lipovich L, McCouch SR, et al. Diversity of microsatellites derived from genomic libraries and GenBank sequence in rice Oryza sativa L. Theor App Genet. 2000;100:713-22.

45. Liewlaksaneeyanawin C, Ritland C, El-Kassaby Y, Ritland K. Single-copy, species-transferable microsatellite markers developed from loblolly pine ESTs. Theor App Genet. 2004;109:361-9.

46. Ranade S, Lin YC, Zuccolo A, Van de Peer Y, García-Gil M. Comparative in silico analysis of EST-SSRs in angiosperm and gymnosperm tree genera. BMC Plant Biol. 2014;14:1471-2229.

47. Pinosio S, González-Martínez SC, Bagnoli F, Cattonaro F, Grivet D, Marroni F, et al. First insights into the transcriptome and development of new genomic tools of a widespread circum-Mediterranean tree species, Pinus halepensis Mill. Mol Ecol Res. 2014;14:846-56.

48. Cordeiro GM, Casu R, McIntyre CL, Manners JM, Henry RJ. Microsatellite markers from sugarcane Saccharum spp. ESTs cross transferable to erianthus and sorghum. Plant Sci. 2000;160:1115-23.

49. Rungis D, Bérubé Y, Zhang J, Ralph S, Ritland EC, Ellis EB, et al. Robust simple sequence repeat markers for spruce Picea spp. from expressed sequence tags. Theor App Genet. 2004;109:1283-94.

50. Eujayl I, Sledge MK, Wang L, May GD, Chekhovskiy K, Zwonitzer JC, et al. Medicago truncatula EST-SSRs reveal cross-species genetic markers for Medicago spp. Theor App Genet. 2004;108:414-22.

51. Wöhrmann T, Weising K. In silico mining for simple sequence repeat loci in a pineapple expressed sequence tag database and cross-species amplification of EST-SSR markers across Bromeliaceae. Theor App Genet. 2011:123:635-47.

52. Russell J, Booth A, Fuller J, Harrower B, Hedley P, Machray G, et al. A comparison of sequence-based polymorphism and haplotype content in transcribed and anonymous regions of the barley genome. Genome. 2004;47:389-98.

53. Conne L, Stutz A, Vasalli JD. The $3^{\prime}$ untranslated region of messenger RNA, A molecular "hotspot" for pathology? Nat Med. 2000;6:637-41.

\section{Submit your next manuscript to BioMed Central and take full advantage of:}

- Convenient online submission

- Thorough peer review

- No space constraints or color figure charges

- Immediate publication on acceptance

- Inclusion in PubMed, CAS, Scopus and Google Scholar

- Research which is freely available for redistribution 